\title{
Is Concomitant Mitral Regurgitation With Severe Aortic Stenosis Benign or Malignant?
}

\author{
Hiroyuki Kiriyama, MD; Masao Daimon, MD
}

$\mathbf{M}$ oderate/severe mitral regurgitation (MR) is a common coexistent disorder in patients with severe aortic stenosis (AS). Because MR may develop in the presence of high ventricular pressure due to severe AS, the frequency of significant MR increases as the severity of AS increases. Therefore, assessment for significant MR complicated with severe AS in need of invasive treatment is never a negligible issue in daily practice. Previous studies $^{1-7}$ have examined the effect of significant MR concomitant with severe AS on mortality, mainly in patients undergoing invasive procedures such as surgical aortic valve replacement (SAVR) or transcatheter aortic valve replacement (TAVR). Most studies, including meta-analyses, focusing on SAVR have shown increased mortality in patients with significant MR compared with those without MR.1-4

\section{Article p 427}

However, mitral valve (MV) interventions were not or rarely performed in those studies. Increased mortality was also noted in patients with significant MR who underwent TAVR compared with those without $\mathrm{MR}^{5-7}$ in whom no MV interventions were done. Thus, due to the lack of sufficient interventions for concomitant MR in patients with severe AS reported in the published literature, its effect in severe AS remains elusive.

In this issue of the Journal, Murai and colleagues ${ }^{8}$ demonstrate that concomitant moderate/severe MR is not independently associated with a high risk of the composite endpoint of heart failure hospitalization and aortic valve-

\section{Concomitant moderate/severe MR in patients with severe AS}

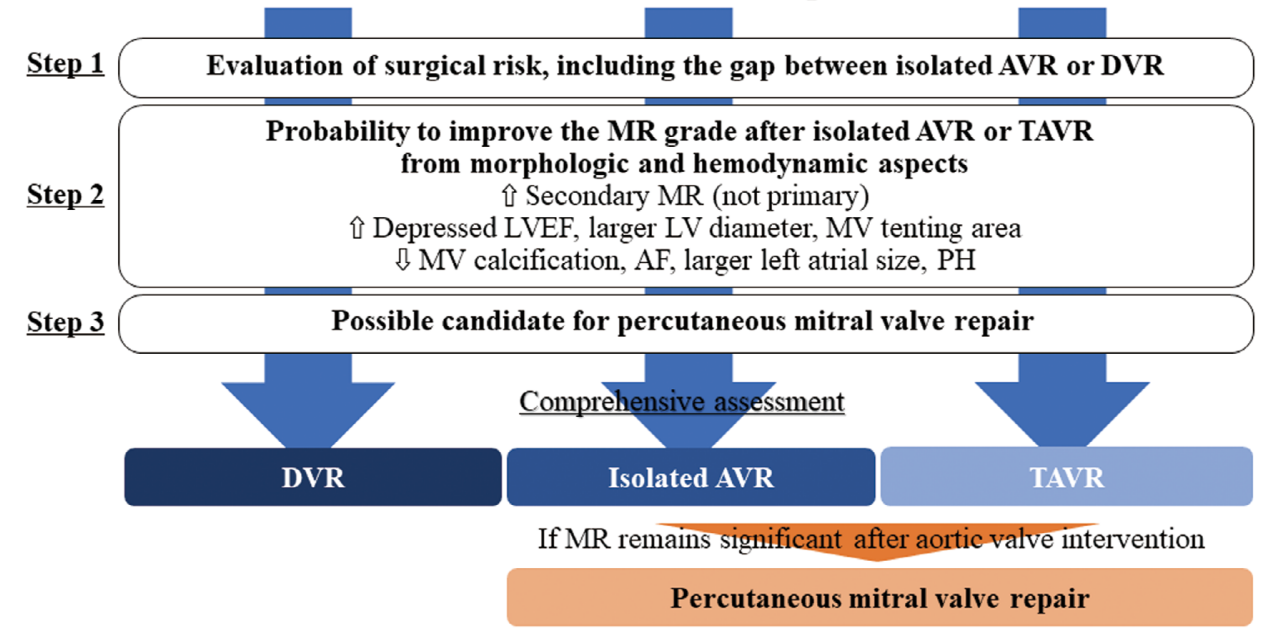

Figure. Strategy for decision making regarding concomitant mitral regurgitation (MR) in patients with severe aortic stenosis (AS). $\mathrm{AF}$, atrial fibrillation; AVR, aortic valve replacement; DVR, double-valve replacement; LVEF, left ventricular ejection fraction; MV, mitral valve; $\mathrm{PH}$, pulmonary hypertension; TAVR, transcatheter aortic valve replacement.

The opinions expressed in this article are not necessarily those of the editors or of the Japanese Circulation Society.

Received July 5, 2021; accepted July 5, 2021; J-STAGE Advance Publication released online August 21, 2021

Department of Cardiovascular Medicine (H.K., M.D.), Department of Clinical Laboratory (M.D.), The University of Tokyo, Tokyo, Japan

Mailing address: Masao Daimon, MD, Department of Cardiovascular Medicine, The University of Tokyo, 7-3-1 Hongo, Bunkyo-ku,

Tokyo, 113-8655, Japan. E-mail: daimon@muf.biglobe.ne.jp

All rights are reserved to the Japanese Circulation Society. For permissions, please e-mail: cj@j-circ.or.jp

ISSN-1346-9843 
related death, regardless of the initial treatment strategy, based on a subanalysis of the CURRENT AS Registry (Contemporary outcomes after sURgery and medical tREatmeNT in patients with severe Aortic Stenosis), one of the largest retrospective studies of AS in Japan. In their report, $19 \%$ of patients who underwent an initial aortic valve replacement (AVR) strategy and $20 \%$ of those who underwent a conservative strategy had moderate/severe MR. In the multivariable analysis, moderate/severe MR was not independently associated with a higher risk of the primary outcome with either the initial AVR strategy (hazard ratio [HR] 1.11, 95\% confidence interval [CI] 0.67-1.83, P=0.69) or the conservative strategy (HR $1.13,95 \%$ CI $0.93-1.37$, $\mathrm{P}=1.22$ ). It is noteworthy that this study showed that preoperative significant MR was not independently associated with an increased risk of heart failure hospitalization, aortic valve-related death, all-cause death, or cardiovascular death, inconsistent with previous studies. ${ }^{1-4}$ The major difference between this study and previous ones is whether the MV intervention was performed in the initial phase. In this cohort, the MV strategy was decided at the discretion of the attending physician, and $38 \%$ of patients with significant MR in the initial AVR group underwent concomitant MV surgery. This proportion was much higher than in the PARTNER SAVR cohort $(5.8 \%) .{ }^{3}$ The current study results suggested that adequate assessments by and decisions of the attending physician for the MV in real-world clinical settings might offset the adverse effects of concomitant moderate/ severe MR, and reconfirmed the utmost importance of optimal decision making for patients with severe AS and concomitant MR, including decisions on MV surgery.

What is the optimal MV strategy in patients with moderate/severe MR concomitant with severe AS? Surgery for MR in conjunction with AVR can significantly and sustainably reduce the MR grade, although the additional risk of manipulating the MV can be problematic in specific populations. Further, the severity of MR should be evaluated in these patients, because AVR itself can often ameliorate the MR grade by releasing the high ventricular pressure. A meta-analysis by Harling and colleagues found that the MR grade improved in more than half of patients who underwent AVR.4 A much greater positive effect of AVR on MR has been seen in patients with secondary MR vs. primary MR. ${ }^{4}$ Also, it has been reported that depressed left ventricular (LV) ejection fraction, larger LV diameter, and MV tenting area may be associated with better improvement of MR; in contrast, calcific degeneration of the MV, atrial fibrillation, large left atrial size and pulmonary arterial hypertension may lead to a lack of improvement in

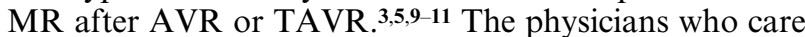
for these patients should evaluate the further surgical risk of adding MV surgery and whether MR will improve with an isolated aortic valve intervention from both the morphologic and hemodynamic aspect, including the origin of MR (primary or secondary). Moreover, they should be aware that percutaneous MV repair with the MitraClip currently allows for less invasive intervention. Combined percutaneous aortic and mitral intervention is currently reported to be a solution for high-risk surgical patients with moderate/ severe MR concomitant to severe AS. ${ }^{\mathbf{1 2}}$ MitraClip could be considered following aortic valve intervention if $\mathrm{MR}$ remains significant after isolated AVR or TAVR.

This subanalysis of the CURRENT AS registry ${ }^{\mathbf{2}}$ demonstrated that real-world decision making for concomitant MR in patients with severe AS in Japan can offset the adverse effects of concomitant moderate/severe MR, highlighting the importance of determining the optimal strategy for moderate/severe MR concomitant with severe AS. We should take the following into account: (1) accurate evaluation of surgical risk, including the gap in surgical risk between singleand double-valve intervention (isolated AVR/double-valve surgery/TAVR), (2) the probability of improving the MR grade after isolated AVR or TAVR from the morphologic and hemodynamic aspects, and (3) whether the patient is a candidate for percutaneous MV repair if significant MR is observed after isolated AVR or TAVR (Figure).

\section{Disclosures}

M.D. is a member of Circulation Journal's Editorial Team. H.K. has no conflicts of interest to disclose.

\section{Conflict of Interest / IRB Information}

None.

\section{References}

1. Barreiro CJ, Patel ND, Fitton TP, Williams JA, Bonde PN, Chan V, et al. Aortic valve replacement and concomitant mitral valve regurgitation in the elderly: Impact on survival and functional outcome. Circulation 2005; 112(Suppl): I443-I447.

2. Ruel M, Kapila V, Price J, Kulik A, Burwash IG, Mesana TG. Natural history and predictors of outcome in patients with concomitant functional mitral regurgitation at the time of aortic valve replacement. Circulation 2006; 114(Suppl): I541 - I546.

3. Barbanti M, Webb JG, Hahn RT, Feldman T, Boone RH, Smith $\mathrm{CR}$, et al. Impact of preoperative moderate/severe mitral regurgitation on 2-year outcome after transcatheter and surgical aortic valve replacement: Insight from the Placement of Aortic Transcatheter Valve (PARTNER) Trial Cohort A. Circulation 2013; 128: $2776-2784$.

4. Harling L, Saso S, Jarral OA, Kourliouros A, Kidher E, Athanasiou T. Aortic valve replacement for aortic stenosis in patients with concomitant mitral regurgitation: Should the mitral valve be dealt with? Eur J Cardiothorac Surg 2011; 40: 1087-1096.

5. Toggweiler S, Boone RH, Rodes-Cabau J, Humphries KH, Lee $M$, Nombela-Franco L, et al. Transcatheter aortic valve replacement: Outcomes of patients with moderate or severe mitral regurgitation. J Am Coll Cardiol 2012; 59: 2068-2074.

6. Bedogni F, Latib A, De Marco F, Agnifili M, Oreglia J, Pizzocri $\mathrm{S}$, et al. Interplay between mitral regurgitation and transcatheter aortic valve replacement with the CoreValve Revalving System: A multicenter registry. Circulation 2013; 128: 2145-2153.

7. Nombela-Franco L, Eltchaninoff H, Zahn R, Testa L, Leon MB, Trillo-Nouche R, et al. Clinical impact and evolution of mitral regurgitation following transcatheter aortic valve replacement: A meta-analysis. Heart 2015; 101: 1395-1405.

8. Murai R, Kawase Y, Taniguchi T, Morimoto T, Kadota K, Ohya M, et al; on behalf of the CURRENT AS Registry Investigators. Concomitant mitral regurgitation in severe aortic stenosis: A report from the CURRENT AS Registry. Circ J 2022; 86: $427-437$.

9. Tzikas A, Piazza N, van Dalen BM, Schultz C, Geleijnse ML, van Geuns RJ, et al. Changes in mitral regurgitation after transcatheter aortic valve implantation. Catheter Cardiovasc Interv 2010; 75: $43-49$.

10. Matsumura Y, Gillinov AM, Toyono M, Oe H, Yamano T, Takasaki K, et al. Echocardiographic predictors for persistent functional mitral regurgitation after aortic valve replacement in patients with aortic valve stenosis. Am J Cardiol 2010; 106: $701-706$.

11. Tassan-Mangina S, Metz D, Nazeyllas P, Torossian F, Pop C, Bertrand $\mathbf{J}$, et al. Factors determining early improvement in mitral regurgitation after aortic valve replacement for aortic valve stenosis: A transthoracic and transesophageal prospective study. Clin Cardiol 2003; 26: 127-131.

12. Okada A, Kanzaki H, Amaki M, Kataoka Y, Miyamoto K, Hamatani $Y$, et al. Successful treatment of mitral regurgitation after transapical transcatheter aortic valve implantation by percutaneous edge-to-edge mitral valve repair (MitraClip ${ }^{\circledR}$ ): The first combination therapy performed in Japan. Intern Med 2018; 57: $1105-1109$. 